\title{
Reports from recent evidence-based conferences
}

\author{
1st International Conference on EBD - November 8-9 - Atlanta, Georgia, USA
}

The 1st International Conference on Evidence-Based Dentistry (EBD) was recently held in Atlanta, Georgia. Over 200 delegates from 12 countries representing many significant stakeholders, such as: clinicians, researchers, academics, lawyers, statisticians, ethicists, regulatory bodies, product manufacturers and private insurance companies, attended this 2-day conference.

Dr Jim Bader began the conference by trying to answer the simple question: Is EBD a new word for an old concept? He argued that what makes EBD a new concept is that for the first time, an attempt is being made to objectively synthesize all current available information for the sake of optimizing the efficiency and efficacy of clinical practice.

Susan Sutherland followed Dr Bader by reviewing the concept of Systematic Reviews (SR). This process differs greatly from the traditional expert reviews, in that SR's follow a standard and rigorous scientific methodology that objectively gathers and critically appraises all sources of information available. Professor Kay Dickersin reminded the audience that even SR's can be subjected to the bias that research with positive outcomes are more likely to be published and thus gathered in a systematic search.

Dr Hodge then reviewed the methods presently used to assess the quality of the literature. Although no single method seemed to be favorable he tended to put more credence on the reliability of "component assessment methods" as compared to "quantitative scale methods".

The concurrent afternoon sessions dealing with the perceptive of EBD to the dental product development and the private dental insurance provider resulted in much discussion and debate amongst the delegates.

Another session presented by William Giannobile, James Summitt, David Cagna and William Shaw summarized "what evidence" is currently available in the field of periodontics, the management of initial carious lesion, prosthodontics and orthodontics respectively. The presenters did not hide the fact that there is a dearth of good quality evidence available for them to make significant conclusion from. But moderator Dr James Bader urged the audience not to become "EBD elitist" by condemning all data generated short of the "gold standard" of the RCT methodology. Random Controlled Trials are not always feasible in dentistry. He suggested that a better way of extracting valid information from such so-called "inferior quality" studies need to be developed by the critical appraisers.

A highlight of the conference was Dr Phillip Hujoel who introduced a four-level scale to assess clinical significance. This should not be confused with levels of clinical evidence or statistical significance. In other words, how should clinicians interpret clinical significance in the face of a well-designed clinical study that, may or may not, have statistical significance?

- Level 1 of Clinical Significance - There exists a "tangible benefit" with a "large treatment effect". At this level, statistical significance is irrelevant because the difference and thus the benefit (or harm) are so obvious from the study that the results "hit you on the head like a two-by-four block of wood". An example of this would be the clinical findings of osseointegration dental implants in human mandibles. The overwhelming success rate of over $90 \%$ is obvious and does not necessarily need the support of statistical analysis.

- Level 2 of Clinical Significance - There exists a "tangible benefit" with a "small treatment effect". In such studies, statistical analysis is needed to support any difference in any mild benefit measured. An example of this would be the benefit of occlusal splint on TMD. Studies may show a small but measurable benefit that nevertheless generates a statistically significant outcome.

- Level 3 of Clinical Significance - A "large significant therapeutic effect on the surrogate end point" (i.e. dental varnish that drastically reduces oral strep. Mutans levels) but no or questionable evidence of a "tangible clinical benefit" (ie lower caries levels).

- Level 4 of Clinical Significance - A "small to moderate therapeutic effect on the surrogate end-point" (ie, Listerine reducing gingival index) with no or questionable evidence of a "tangible clinical benefit" (i.e., lower future tooth loss)

Statistical analysis helps to determine the reliability of the data in a clinical study, especially when the measure outcome is mild or moderate (Level 2 and 4 of clinical significance) but it does not determine clinical significance. Clinical significance is a subjective matter determined by the patient in consultation with the dentist.

Concurrent sessions in the role of the educator in EBD and cutting edge meta-analysis methodology were presented by expert speakers in their fields.

The Ethical and Legal obligations in the use of EBD were presented by Dr Mary McNally (a dental ethicist) and Ms. Elaine Gibson (a lawyer). The ethical role of EBD must be assessed in the context on the major stakeholder, including the patient. Ms. Gibson believes that the positive implication of EBD to the law is that better clinical evidence means better clinical and legal decisions.

The final session reviewed the use of evidence in dental product advertising in the lay media. Representatives from ColgatePalmolive, a major American television network's advertising department (ABC Inc.) and the National Advertising Division (an industry watch-dog which reviews the validity of national advertising) were on the panel to discuss their ethical duty to using evidence in the way they deliver their message in the media. Dr Bader commented that there may be a difference in the way evidence is used for commercial use. In EBD, evidence is synthesized by many sources in the hope of getting the overall truth while advertisers seek only the evidence that supports their claims, regardless of quantity or quality of the evidence, an approach that harks back to the traditional approach to evidence rather than the one EBD espouses.

The conference ended with a keynote speech from Dr Roman Jaeschke, a professor from McMaster's University and a pioneer in the EBM movement. He remarked that the power of evidence-based practice is in medical decision-making. The patients in consultation with the clinician make the decision using clinical evidence.

Many dimensions of EBD were introduced and successfully presented in a concise and clear manner. The meeting was also 
well organized and much credit for the success of this 1st International Conference on EBD must go to Dr Debora Matthews and Dr Mike Newman.

I look forward to the publication of the Proceedings of the conference in a forthcoming issue of The Journal of Evidence-based Dental Practice, one of the co-sponsors of the Conference, and future international conferences on EBD. It enables participants to analyze and discuss applicable clinical summaries, conclusions and guidelines. Another positive aspect of the conference was the ample opportunity to ask questions during the session's question period. Also, during the coffee breaks, participants were able to debate and express their points of view. The benefit of EBD is primarily intended to benefit the "dentist in the trenches" with the objective being better oral health outcomes. As a full-time practicing dentist, I left this conference feeling I was a contributor and a member of the EBD movement seeking to implement EBD into clinical practice.

\section{Ben Balevi}

Dental Practitioner, Madoc, Ontario, Canada

Evidence-Based Dentistry (2004) 5, 18-19. doi:10.1038/sj.ebd.6400229

\section{Odontologia Baseada Evidência - 17-24 November — Brazil}

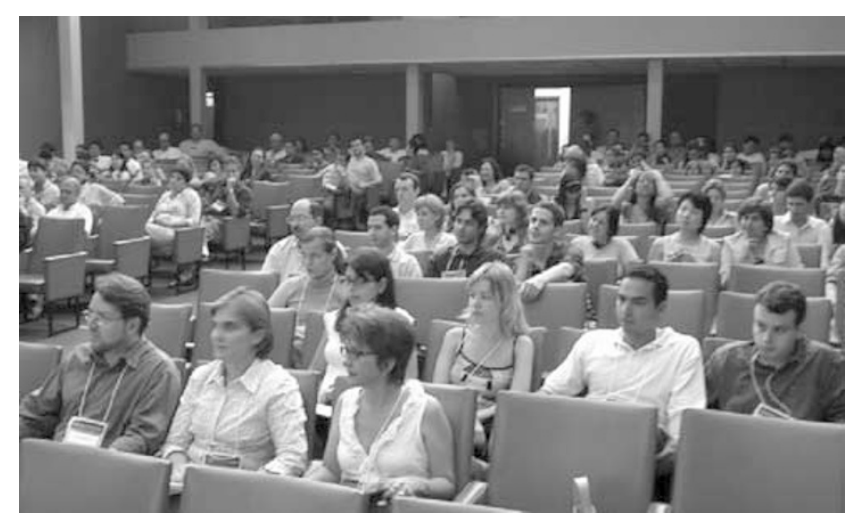

Following the success of the evidence-based dentistry conference in Atlanta (Georgia, USA), I am pleased to report on two similarly successful conferences and workshops held in Brazil in November 2003. The events were organised following the attendance of the Maria Navarro Dean of the dental faculty from the University of São Paulo at Bauru, and Professor Aronita Rosenblatt from the dental faculty at the University of Pernambuco at the Atlanta meeting.

The Bauru event was a 2-day conference followed by a 3-day workshop and the Recife event a half-day conference followed by a day of workshops. The majority of the sessions over the week were presented by Debora Matthews and me; Richard Niederman had to leave early because of family commitments and, unfortunately, Valeria Marinho - who many will recall from her excellent and extensive series of Cochrane topical fluoride reviews - was unwell. But despite these minor setbacks, and the temperatures in the $30^{\circ} \mathrm{C}$ range, both events were very well-attended and attracted over 200 participants in all. These included both staff and students from the two dental schools along with staff from other medical disciplines.

The team outlined the evidence-based approach and the underlying skills of question formulation, searching for evidence and critical appraisal. These were then developed in interactive workshops looking at appraisal techniques for systematic reviews, randomised controlled trials, cohort studies, diagnostic tests and guidelines. The whole programme was presented with simultaneous translation and happily this did not prevent enthusiastic participation, with much interaction between the speakers and the audience. It was refreshing to see such a level of enthusiasm for this approach and the determination of some of the staff and students to understand and pursue the evidence-based approach in their dental schools in Brazil.

The hospitality offered by our hosts was also outstanding: we thank them and also the Brazilian Government which helped fund the conferences.

\section{Derek Richards \\ Centre for Evidence-based Dentistry, Oxford, UK}

Evidence-Based Dentistry (2004) 5, 19. doi:10.1038/sj.ebd.6400245 